\title{
Verteporfin suppresses osteosarcoma progression by targeting the Hippo signaling pathway
}

\author{
XIANLIANG YANG ${ }^{1,2}$, YOUJIA XU ${ }^{1}$, CHAO JIANG ${ }^{2}$, \\ ZIPING $\mathrm{MA}^{2}$ and LINGUANG JIN ${ }^{2}$ \\ ${ }^{1}$ Department of Orthopedics, The Second Affiliated Hospital of Soochow University, Suzhou, Jiangsu 215004; \\ ${ }^{2}$ Department of Orthopedics, The First People's Hospital of Wenling Hospital, Wenling, Zhejiang 317500, P.R. China
}

Received November 12, 2020; Accepted July 1, 2021

DOI: $10.3892 / 01.2021 .12985$

\begin{abstract}
Verteporfin (VP) is a specific inhibitor of yes-associated protein 1 (YAP1) that suppresses tumor progression by inhibiting YAP1 expression. The present study aimed to determine the inhibitory effect of VP on osteosarcoma and the underlying mechanism of its anticancer effects. Cell viability, cell cycle and apoptosis and cell migration and invasion were analyzed using the MTT assay, flow cytometry, wound healing assay and Transwell assay, respectively. Expressions of YAP1 and TEA domain transcription factor 1 (TEAD1) were measured using reverse transcription-quantitative PCR and western blotting, while their interaction was identified by the co-immunoprecipitation assay. In vivo mouse xenograft experiments were performed to evaluate the effect of VP on osteosarcoma growth. The results demonstrated that YAP1 and TEAD1 were highly expressed in osteosarcoma cells and tissues, whereas VP significantly downregulated the expression levels of YAP1 and TEAD1 in the osteosarcoma cell line Saos-2 compared with those in untreated control cells. In addition, compared with those in the control group, VP suppressed the viability, migration and invasion, induced cell cycle arrest in the G1 phase and promoted apoptosis in Saos-2 cells. In addition, VP inhibited mouse xenograft tumor growth in vivo compared with that observed in the control group. Notably, VP downregulated the levels of CYR61 expression in Saos-2 cells, whereas CYR61 overexpression mitigated the inhibitory effects of VP on osteosarcoma cells, as indicated by the increased viability and reduced apoptotic rates in Saos-2 cells overexpressing CYR61 compared with those in the control group. In summary, VP suppressed osteosarcoma by downregulating the expression of YAP1 and TEAD1. Additionally,
\end{abstract}

Correspondence to: Dr Youjia Xu, Department of Orthopedics, The Second Affiliated Hospital of Soochow University, 1055 Sanxiang Road, Suzhou, Jiangsu 215004, P.R. China

E-mail: xuyoujia@suda.edu.cn

Key words: osteosarcoma, yes-associated protein 1, TEA domain transcription factor 1, verteporfin, tumorigenesis, cysteine-rich protein 61
CYR61 may mediate the effects of VP on osteosarcoma progression.

\section{Introduction}

Osteosarcoma is a typical primary bone malignancy that occurs mainly in children and adolescents aged 10-20 years (1). In Europe, the incidence of osteosarcoma is between 2 and 5 new diagnoses per 1 million people per year, while in the USA, 800 subjects are diagnosed with osteosarcoma per year $(2,3)$. At present, the standard treatments for osteosarcoma include surgery and neoadjuvant chemotherapy, such as methotrexate, cisplatin and doxorubicin (4). It has been reported that cabozantinib can directly suppress osteosarcoma cell proliferation by inhibiting the ERK and Akt signaling pathways (5). Despite notable progresses in the treatment of osteosarcoma, the 5-year overall survival rate of patients with osteosarcoma remains only $20-30 \%$ due to its susceptibility to local invasion and early metastasis, with $\leq 25 \%$ of patients developing lung metastasis (6). Han et al (7) have suggested that the upregulation of c-Myc is implicated in the invasion of osteosarcoma cells by activating the MEK-ERK pathway. Various target genes of microRNAs (miRs) participate in the intracellular signaling pathways of osteosarcoma, including the c-Met, Notch, Ras/p21, MAPK, Wnt and Jun/Fos pathways (8). For example, long non-coding RNA plasmacytoma variant translocation 1 downregulates miR-152 expression to activate the c-Met/PI3K/AKT signaling pathway, facilitating chemoresistance of osteosarcoma cells to gemcitabine (9). Therefore, the development of new effective therapeutic strategies for osteosarcoma is urgently required.

As an evolutionarily conserved developmental network, the Hippo signaling pathway impedes tumorigenesis through activating large tumor suppressor kinases and controlling the downstream effectors yes-associated protein (YAP) and transcriptional co-activator with PDZ-binding motif (TAZ) (10). When the Hippo pathway is inactive, YAP and TAZ are dephosphorylated and interact with TEA domain transcription factors (TEADs) to regulate cell viability and apoptosis $(11,12)$. YAP1, which is involved in the Hippo signaling pathway, has been reported to be upregulated in osteosarcoma and associated with a poor prognosis (13). Several oncogenic pathways feed into the Hippo signaling pathway in a YAP1-dependent 
manner, such as Wnt/ $\beta$-catenin, PI3K/AKT, MAPK and JAK/STAT signaling pathways (14). YAP1-mediated oncogenic effects are dependent on TEADs (15). TEADs can promote tumor progression by regulating the expression levels of tumor-promoting genes, including dynamin 3 (DYN3), prostaglandin-endoperoxide synthase 2 (PTGS2), lysyl oxidase (LOX), brain-derived neurotrophic factor (BDNF), connective-tissue growth factor (CTGF) and cysteine-rich protein 61 (CYR61) (12).

As a specific inhibitor of YAP1, verteporfin (VP) can be used as a drug that promotes phototherapy in the treatment of neovascular macular degeneration (16). YAP1 in trabecular meshwork cells has been demonstrated to be suppressed by VP without light stimulation $(17,18)$. Pan et al (19) have demonstrated that VP reverses paclitaxel resistance by specifically inhibiting YAP1 expression in patients with colon cancer. Although the roles of VP have been documented in various types of malignant tumors, its function in osteosarcoma progression needs to be further investigated.

The present study aimed to investigate the effects of VP on osteosarcoma and the underlying mechanism of its action using patient-derived osteosarcoma tissues, osteosarcoma cell lines and a mouse model. We hypothesized that VP may inhibit the tumorigenesis of osteosarcoma by blocking the binding of YAP1 to TEAD1 and suppressing the expression of the downstream targets of TEAD1. This may provide important guidance for the treatment of osteosarcoma.

\section{Materials and methods}

Patient samples and cell culture. Osteosarcoma specimens and paired tumor-adjacent tissues were collected from eight patients aged $\geq 16$ years with bone and joint pain in the First People's Hospital of Wenling (Wenling, China). Among the patients, three were male and five were female, with a median age of 37 years and age range 18-56 years. All patients were diagnosed with stage IIA osteosarcoma in the distal femur and proximal tibia by $\mathrm{CT}$ scans, magnetic resonance imaging and bone scans (20). The patients underwent surgery, and osteosarcoma was pathologically confirmed. Signed written informed consent was obtained from all patients or guardians and the procedure was approved by the Ethics Committee of the First People's Hospital of Wenling (approval no. KY-2019-2001-01).

The normal human osteoblast cell line NHOst and osteosarcoma cell lines U2OS (cat. no. HTB-96), HOS and Saos-2 were purchased from ATCC. NHOst, U2OS and Saos-2 cell lines were cultured in McCoy's 5A medium containing 15\% FBS (both Gibco; Thermo Fisher Scientific Inc.) and 1\% penicillin-streptomycin-glutamine to $70-80 \%$ confluency. The HOS cell line was cultured in 10\% FBS-containing Eagle's minimum essential medium (Sigma-Aldrich; Merck KGaA) to $80 \%$ confluency. All cell lines were cultured at $37^{\circ} \mathrm{C}$ with $5 \% \mathrm{CO}_{2}$ in a humidified incubator and passaged into $\mathrm{T} 75$ flasks (passage three). Saos-2 cells were cultured with VP (HY-B0146; MedChemExpress) at 0,2.5 and $5 \mu \mathrm{M}$ at room temperature for $24 \mathrm{~h}$.

Reverse transcription-quantitative (RT-q) PCR. Total RNA was extracted from osteosarcoma and paired tumor-adjacent tissues, as well as NHOst, U2OS, HOS and Saos-2 cells by
TRIzol ${ }^{\circledR}$ reagent (cat. no. 15596018; Invitrogen; Thermo Fisher Scientific, Inc.). The total RNA was reverse-transcribed to cDNA using a PrimeScript ${ }^{\mathrm{TM}}$ RT reagent kit (cat. no. RR037Q; Takara Bio, Inc.). Briefly, $10 \mu \mathrm{l}$ of the reverse transcription system containing $\sim 325 \mathrm{ng}$ RNA (50 ng $/ \mu \mathrm{l} ; 6.5 \mu \mathrm{l})$ was used, and the reverse transcription was performed at $37^{\circ} \mathrm{C}$ for $30 \mathrm{~min}$, followed by $85^{\circ} \mathrm{C}$ for $5 \mathrm{sec}$ and $4^{\circ} \mathrm{C}$ for maintenance. The cDNA was diluted with $50 \mu 1$ double-distilled water, and mRNA expression was measured using a SYBR ${ }^{\circledR}$ Premix Ex Taq ${ }^{\mathrm{TM}}$ kit (cat. no. RR42LR; Takara Bio, Inc.). The thermocycling condition used were as follows: $95^{\circ} \mathrm{C}$ for $10 \mathrm{~min}$, followed by 40 cycles of $95^{\circ} \mathrm{C}$ for $15 \mathrm{sec}$ and $60^{\circ} \mathrm{C}$ for $45 \mathrm{sec}$, then $95^{\circ} \mathrm{C}$ for $15 \mathrm{sec}, 60^{\circ} \mathrm{C}$ for $1 \mathrm{~min}, 95^{\circ} \mathrm{C}$ for $15 \mathrm{sec}$ and $60^{\circ} \mathrm{C}$ for $15 \mathrm{sec}$. PCR samples were analyzed with a real-time PCR system (Thermo Fisher Scientific Inc.). The expression of YAP1, TEAD1, DYN3, PTGS2, LOX, BDNF, CTGF and CYR61 mRNA was normalized to that of GAPDH and calculated using the $2^{-\Delta \Delta C q}$ method (21). The primer sequences were presented in Table I.

Western blot analysis. Total protein was extracted from osteosarcoma and paired tumor-adjacent tissues, as well as NHOst, U2OS, HOS and Saos-2 cells with RIPA lysis and extraction buffer (cat. no. 89900; Thermo Fisher Scientific, Inc.), followed by centrifugation at $14,000 \mathrm{xg}$ for $15 \mathrm{~min}$ at $4^{\circ} \mathrm{C}$. The protein concentrations were measured using a BCA protein assay kit (Thermo Fisher Scientific, Inc.). Proteins ( $25 \mu \mathrm{g}$ per lane) were separated by SDS-PAGE (12\% resolving gel and 5\% stacking gel) and transferred to a PVDF membrane. Following blocking with skimmed milk powder in TBS containing $0.1 \%$ Tween-20 (TBST) for $1 \mathrm{~h}$ at room temperature, the membrane was incubated overnight at $4^{\circ} \mathrm{C}$ with each of the following primary antibodies (1:1,000 dilution): anti-GAPDH (rabbit mAb; cat. no. 5174; Cell Signaling Technology, Inc.), anti-TEAD1 (rabbit mAb; cat. no. 12292; Cell Signaling Technology, Inc.) and anti-YAP1 (rabbit mAb; cat. no. ab52771; Abcam). GAPDH was used as a loading control. After being washed twice in TBST, the membrane was incubated with a horseradish peroxidase-conjugated goat anti-rabbit antibody (1:1,000; cat. no. ab6721; Abcam) for $2 \mathrm{~h}$ at room temperature. The protein bands were visualized using an enhanced chemiluminescence substrate (cat. no. 32109; Thermo Fisher Scientific, Inc.), and the bands were semi-quantified by ImageJ software v.1.8.0 (National Institutes of Health).

Transwell assay. Transwell assay was performed to assess the effects of VP on cell invasion. The Transwell filter chamber was precoated with Matrigel $(50 \mathrm{mg} / \mathrm{l}$; BD Biosciences) at a ratio of $1: 8$ at $4^{\circ} \mathrm{C}$ for $30 \mathrm{~min}$. Saos-2 cells were cultured in FBS-free Dulbecco's modified Eagle's medium (DMEM; Sigma-Aldrich; Merck KGaA) for $24 \mathrm{~h}$, washed twice with PBS and resuspended in the medium to a density to $2 \times 10^{5}$ cells $/ \mathrm{ml}$. A total of $200 \mu \mathrm{l}$ cell suspension per well was added to the upper chamber, and $400 \mu \mathrm{l}$ medium with $15 \%$ FBS was added to the lower chamber in a 24-well plate. Following a 48-h incubation at $37^{\circ} \mathrm{C}$ with $5 \% \mathrm{CO}_{2}$, all fixations were with $4 \%$ formaldehyde solution $(1 \mathrm{ml})$ at room temperature for $10 \mathrm{~min}$. After inhaling the stationary fluid, the cells on the upper surface were removed with cotton swabs, and those adhering to the bottom of the membrane were stained with $0.1 \%$ crystal violet at $37^{\circ} \mathrm{C}$ for 
Table I. Primer sequences.

\begin{tabular}{ll}
\hline Gene & \multicolumn{1}{c}{ Sequences (5'-3') } \\
\hline YAP1 & F: TAGCCCTGCGTAGCCAGTTA \\
& R: TCATGCTTAGTCCACTGTCTGT \\
TEAD1 & F: ATGGAAAGGATGAGTGACTCTGC \\
& R: TCCCACATGGTGGATAGATAGC \\
PTGS2 & F: CTGGCGCTCAGCCATACAG \\
& R: CGCACTTATACTGGTCAAATCCC \\
LOX & F: GCCGACCAAGATATTCCTGGG \\
& R: GCAGGTCATAGTGGCTAAACTC \\
BDNF & F: GGCTTGACATCATTGGCTGAC \\
& R: CATTGGGCCGAACTTTCTGGT \\
FTGF & F: CAGCATGGACGTTCGTCTG \\
& R: AACCACGGTTTGGTCCTTGG \\
CYR61 & F: CTCGCCTTAGTCGTCACCC \\
& R: CGCCGAAGTTGCATTCCAG \\
DYN3 & F: AGTTCGCCTTGAGATTGAAGC \\
& R: CGTGTGGGGAATAGACTCGTAAA \\
GAPDH & F: GGAGCGAGATCCCTCCAAAAT \\
& R: GGCTGTTGTCATACTTCTCATGG
\end{tabular}

YAP1, yes-associated protein 1; TEAD1, TEA domain transcription factor 1; PTGS2, prostaglandin-endoperoxide synthase 2; LOX, lysyl oxidase; BDNF, brain-derived neurotrophic factor; CTGF, connective tissue growth factor; CYR61, cysteine-rich protein 61; DYN3, dynamin 3.

$30 \mathrm{~min}$. The number of invasive cells was counted under a light microscope (Shanghai Cai Kang Optical Instrument Co., Ltd.) (magnification, x100) in 5 randomly selected fields/well.

Wound healing assay. Wound healing assay was performed to determine the impact of VP on cell migration. Saos-2 cells were seeded in 6-well plates and cultured to $80 \%$ confluence. A $1.5-\mathrm{mm}$ wide scratch was created using a $10 \mu \mathrm{l}$ sterile micropipette tip. The cells were washed twice with PBS to remove cellular debris and cultured in serum-free DMEM at $37^{\circ} \mathrm{C}$ for $24 \mathrm{~h}$. The cell migration was observed under a light microscope and the scratches were measured at 24 h by ImageJ software v.1.8.0 (National Institutes of Health).

MTT assay. MTT assay was performed to evaluate cell viability. Saos- 2 cells were seeded into 24 -well plates at a density of $1 \times 10^{5}$ cells per well. Following a 24-h culture, the medium was removed, and the cells were washed with PBS three times. Then, the cells were treated with $2.5 \mu \mathrm{M}$ VP for 24,48 and $72 \mathrm{~h}$ at $37^{\circ} \mathrm{C}$. Subsequently, $50 \mu \mathrm{l}$ MTT solution (Sigma-Aldrich; Merck KGaA) was added to each well for $4 \mathrm{~h}$. A total of $500 \mu$ l dimethyl sulfoxide (Sigma-Aldrich; Merck $\mathrm{KGaA}$ ) was used to dissolve the formazan crystals. Absorbance was measured at $490 \mathrm{~nm}$ using a microplate reader.

Co-immunoprecipitation. Co-immunoprecipitation assay was performed as previously described (22) using a Pierce ${ }^{\mathrm{TM}}$ Co-Immunoprecipitation kit (cat. no. 26149; Thermo Fisher Scientific, Inc.). Briefly, Saos-2 cells were collected, and lysed with immunoprecipitation buffer (50 mM Tris- $\mathrm{HCl} \mathrm{pH} 8.0$, $150 \mathrm{mM} \mathrm{NaCl}, 0.5 \% \mathrm{NP} 40,1 \%$ protease inhibitor cocktail) for $30 \mathrm{~min}$ on ice, followed by centrifugation at $1,1000 \mathrm{x} \mathrm{g}$ for $20 \mathrm{~min}$ at $4^{\circ} \mathrm{C}$. Subsequently, $1 \mathrm{mg}$ protein supernatant was incubated with a YAP1 antibody (dilution, 1:1,000) (rabbit mAb; cat. no. ab52771; Abcam) overnight at $4^{\circ} \mathrm{C}$ and then proG agarose beads were added and incubated for $3 \mathrm{~h}$ with rotation at $4^{\circ} \mathrm{C}$. After washing the beads 3-5 times with immunoprecipitation buffer, the immune complex was collected and analyzed for western blotting as described previously.

Flow cytometry. Saos-2 cells were cultured in an incubator with $5 \% \mathrm{CO}_{2}$ at $37^{\circ} \mathrm{C}$ for $48 \mathrm{~h}$ and rinsed with FACS buffer (PBS containing 0.2\% BSA; Sigma-Aldrich; Merck KGaA). Cells $\left(5 \times 10^{5}\right.$ cells/well) were resuspended in $500 \mu$ l binding buffer and stained with $5 \mu \mathrm{l}$ Annexin V-fluorescein isothiocyanate (cat. no. 556419) and $10 \mu \mathrm{l}$ propidium iodide (PI; cat. no. 556463) (both BD Pharmingen; BD Biosciences) at room temperature for 5-15 min. Flow cytometry (CytoFlex S, Beckman Coulter, Inc.) was performed to determine the apoptotic rates (early + late apoptosis) and the data were quantified using FlowJo v.10.7.1 software (FlowJo, Inc.).

Cell cycle distribution was detected by PI staining. In brief, Saos- 2 cells $\left(5 \times 10^{5}\right.$ cells/well) were fixed with $70 \%$ ethanol overnight at $-20^{\circ} \mathrm{C}$. After washing with PBS three times, cells were incubated with $0.2 \mathrm{mg} / \mathrm{ml}$ RNase, $0.1 \%$ TRITONX-100 and $0.02 \mathrm{mg} / \mathrm{ml} \mathrm{PI}$ at $37^{\circ} \mathrm{C}$ for $30 \mathrm{~min}$. A flow cytometer (CytoFlex S; Beckman Coulter, Inc.) was used to detect red fluorescence and scattered light at an excitation wavelength of $488 \mathrm{~nm}$. The cell cycle analysis was performed using Flow Jo v.10.7.1 software (Flow Jo, Inc.).

Animal experiments. A total of $30 \mathrm{BALB} / \mathrm{c}$ nude mice (age, 4-5 weeks; weight, 15-16 g) used in this study were provided by Wenzhou Medical University (Wenzhou, China) and housed in the specific pathogen free laboratory of Wenzhou Medical University under controlled conditions of $12 \mathrm{~h} \mathrm{light/dark} \mathrm{cycle,}$ with a temperature of $22 \pm 2^{\circ} \mathrm{C}$, a humidity of $50-60 \%$, as well as adequate food and drinking water. The protocols were approved by the Wenzhou Medical University Animal Policy and Welfare Committee (approval no. wydw2019-0616). The suspensions $(150 \mu \mathrm{l})$ of Saos-2 cells $\left(5 \times 10^{7}\right.$ cells/mouse) were subcutaneously injected into the mice. When the tumor volume reached $50-100 \mathrm{~mm}^{3}$, the mice were randomly divided into the control (saline) and VP groups ( $\mathrm{n}=15 \mathrm{mice} / \mathrm{group})$. In the VP group, the mice were intraperitoneally injected with $50 \mathrm{mg}$ VP once every 2 days. Tumor volumes, tumor weights and body weights were measured on days $4,7,11,14,18,21,25$ and 28 post-inoculation. The tumor volume was calculated using the following formula: Volume $=\left(\right.$ length $\mathrm{x}$ width $\left.{ }^{2}\right) / 2$. Animal health and behavior were monitored daily. On day 28 , all mice were euthanized by an intravenous injection with $80 \mathrm{mg} / \mathrm{kg}$ pentobarbital sodium, followed by cervical dislocation. Death was confirmed by observing respiratory and cardiac arrest.

CYR61 overexpression. Saos-2 cells were infected with negative control (pcDNA3.1; empty vector; $1 \mu \mathrm{g} / \mathrm{ml}$; Shaanxi YouBio Technology Co., Ltd.) or CYR61 overexpression plasmid (pcDNA3.1-Cyr61; $1 \mu \mathrm{g} / \mathrm{ml}$, Shaanxi YouBio Technology Co., Ltd.) using Lipofectamine ${ }^{\circledR} 3000$ (Thermo 
A

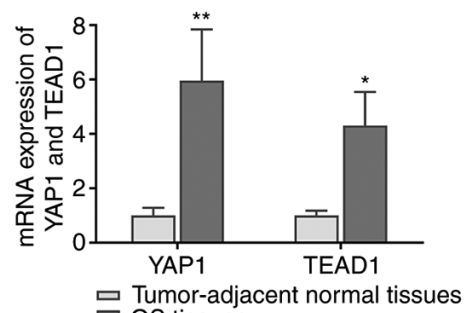

口 Tumor-ad
$\mathrm{B}$

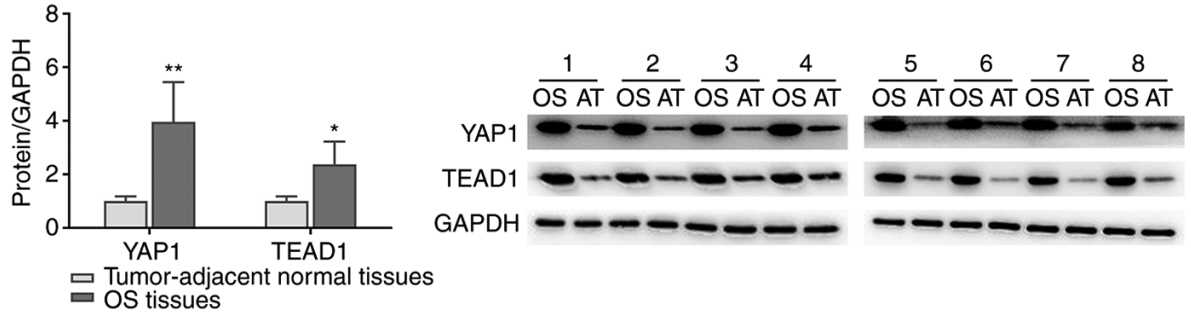

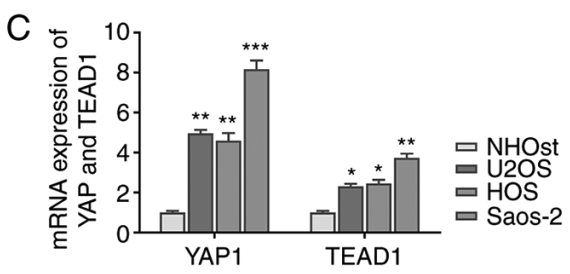
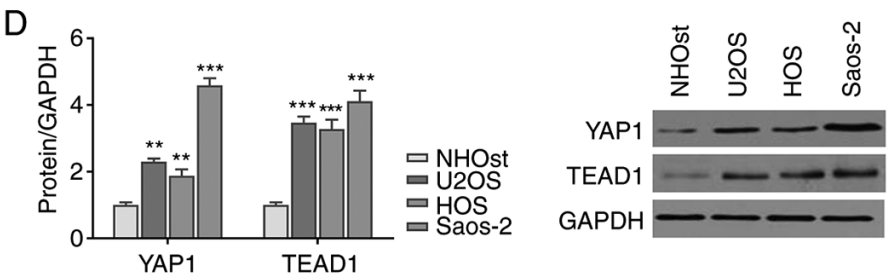

Figure 1. YAP1 and TEAD1 are highly expressed in osteosarcoma tissues and cells. (A and B) The mRNA and protein levels of YAP1 and TEAD1 were detected in osteosarcoma tissues and paired tumor-adjacent normal tissues by (A) RT-qPCR and (B) western blot analysis, respectively. "P $<0.05$ and ${ }^{* *} \mathrm{P}<0.01$ vs. normal tissues. (C) RT-qPCR and (D) western blot analyses were performed to determine the mRNA and protein expression levels of YAP1 and TEAD1 in normal the human osteoblast cell line NHOst and the osteosarcoma cell lines U2OS, HOS and Saos-2. All experiments were performed in triplicate. ${ }^{*} \mathrm{P}<0.05$, ${ }^{* *} \mathrm{P}<0.01$ and ${ }^{* * * *} \mathrm{P}<0.001$ vs. NHOst. YAP1, yes-associated protein 1; TEAD1, TEA domain transcription factor 1; RT-qPCR, reverse transcription-quantitative PCR.

Fisher Scientific, Inc.) according to the manufacturer's instructions for $10 \mathrm{~min}$ at room temperature, and then cultured with $5 \% \mathrm{CO}_{2}$ at $37^{\circ} \mathrm{C}$ for $24 \mathrm{~h}$. Subsequent experiments were performed $48 \mathrm{~h}$ after transfection.

Statistical analysis. Data are presented as the mean \pm standard deviation and were analyzed using GraphPad Prism version 7.0 software (GraphPad Software, Inc.). Comparisons between two groups were analyzed by paired or unpaired Student's t-tests. Statistical differences among multiple groups were determined by one-way ANOVA with Tukey's post hoc test. Two-way ANOVA was used for the analysis of two or more groups over multiple measurements. $\mathrm{P}<0.05$ was considered to indicate a statistically significant difference.

\section{Results}

YAPI and TEADl are highly expressed in osteosarcoma. As presented in Fig. 1A and B, a respective 5.96- and 4.31-fold increase in the mRNA expression levels of YAP1 and TEAD1 was evident in osteosarcoma tissues compared with those in the paired tumor-adjacent normal tissues. Similarly, the protein levels of YAP1 and TEAD1 in osteosarcoma tissues were 3.95and 2.37-fold higher compared with those in the normal tissues, respectively. The expression levels of YAP1 and TEAD1 were further determined in a normal human osteoblast cell line as well as osteosarcoma cell lines. Consistently, the mRNA and protein levels of YAP1 and TEAD1 in osteosarcoma cell lines were significantly higher compared with those in normal the human osteoblast cell line (Fig. 1C and D). Among the osteosarcoma cell lines, Saos-2 cells displayed the highest expression levels of YAP1 and TEAD1; therefore, the Saos-2 cell line was selected for subsequent experiments.

$V P$ impedes the interaction between YAPI and TEAD1. The effects of VP on YAP1 and TEAD1 expression were next evaluated. Western blot analysis was performed on Saos-2 cells treated with various concentrations of VP $(0,2.5$ and $5 \mu \mathrm{M})$ for $24 \mathrm{~h}$. As illustrated in Fig. 2A, VP markedly downregulated the protein expression levels of YAP1 and TEAD1 and the levels appeared to decrease further at a higher dose of VP. Since the binding of YAP1 and TEAD1 induces cell proliferation, differentiation and survival (23), the present study examined whether VP affected the interaction between YAP1 and TEAD1 by co-immunoprecipitation assay. As demonstrated in Fig. 2B, VP weakened the interaction between YAP1 and TEAD1, suggesting that VP may affect Hippo signaling pathway by targeting the formation of the YAP1/TEAD1 complex.

$V P$ regulates the viability, apoptosis and cell cycle progression of Saos-2 osteosarcoma cells. MTT assay and flow cytometry were performed to analyze the viability, apoptosis and cell cycle distribution of Saos-2 cells treated with VP. As presented in Fig. 2C, treatment of Saos-2 cells with VP for $72 \mathrm{~h}$ led to a reduction of the cell viability to $35.1 \%$ of that observed in the control group. Notably, the apoptotic rate was increased from $2.3 \%$ in the control group to $23.5 \%$ in Saos- 2 cells treated with $2.5 \mu \mathrm{M}$ VP (Fig. 2D). In addition, VP treatment resulted in the accumulation of Saos-2 cells in the G1 phase (control, $39.1 \%$ vs. VP, $56.03 \%$ ), accompanied by a reduction in the number of cells in the S and G2 phases (7.27 and 9.66\% in the VP group, respectively), indicating that VP may affect the Saos-2 cell cycle progression (Fig. 2E).

$V P$ inhibits migration and invasion of Saos-2 cells and reduces tumor growth in vivo. Wound healing and Transwell assays were performed to determine the effects of VP on the migration and invasion of Saos-2 cells in vitro, respectively. As illustrated in Fig. 3A and B, significant decreases in cell migration and invasion were observed in Saos- 2 cells treated with VP compared with those in the control cells.

To further evaluate the effects of VP on tumor growth, nude mice were used in the in vivo experiments. As demonstrated in 
A

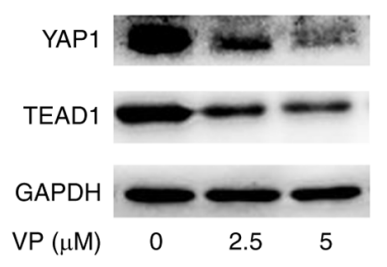

$\mathrm{D}$
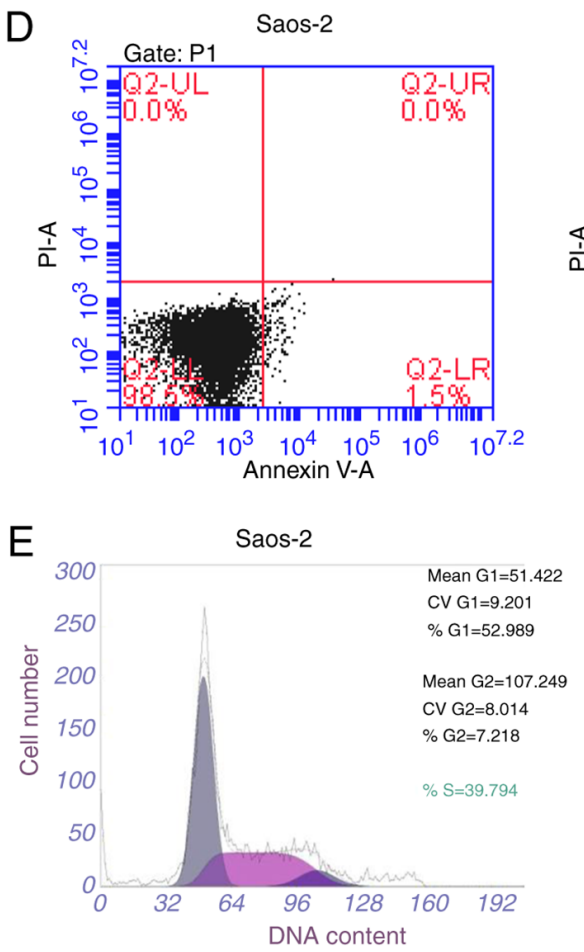

B
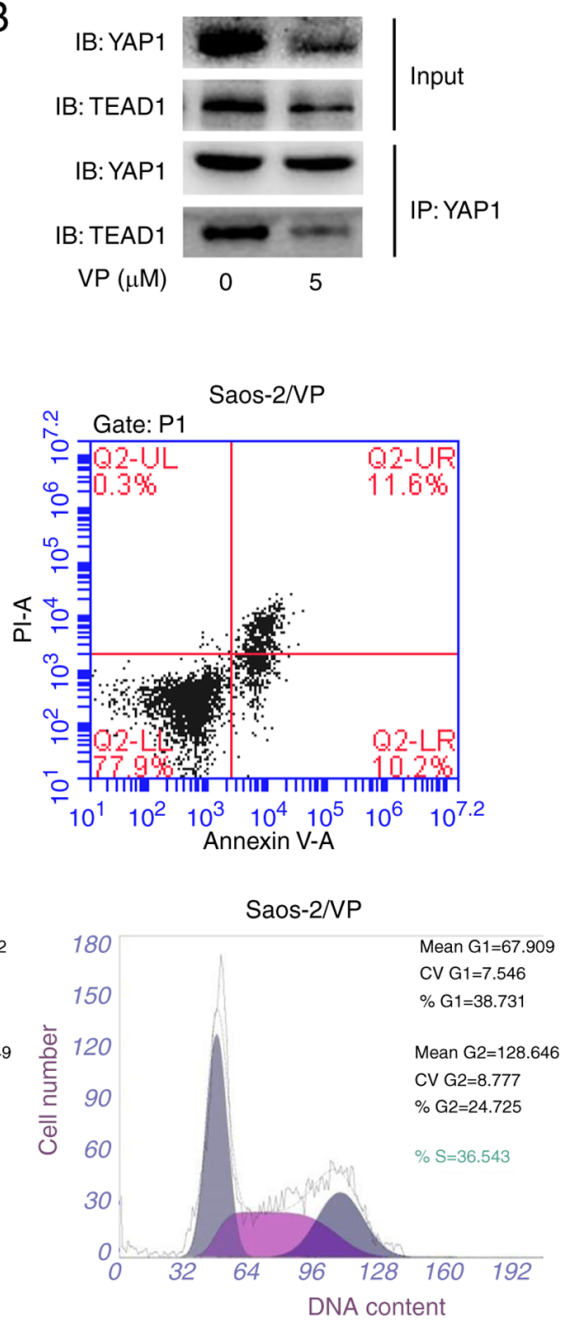
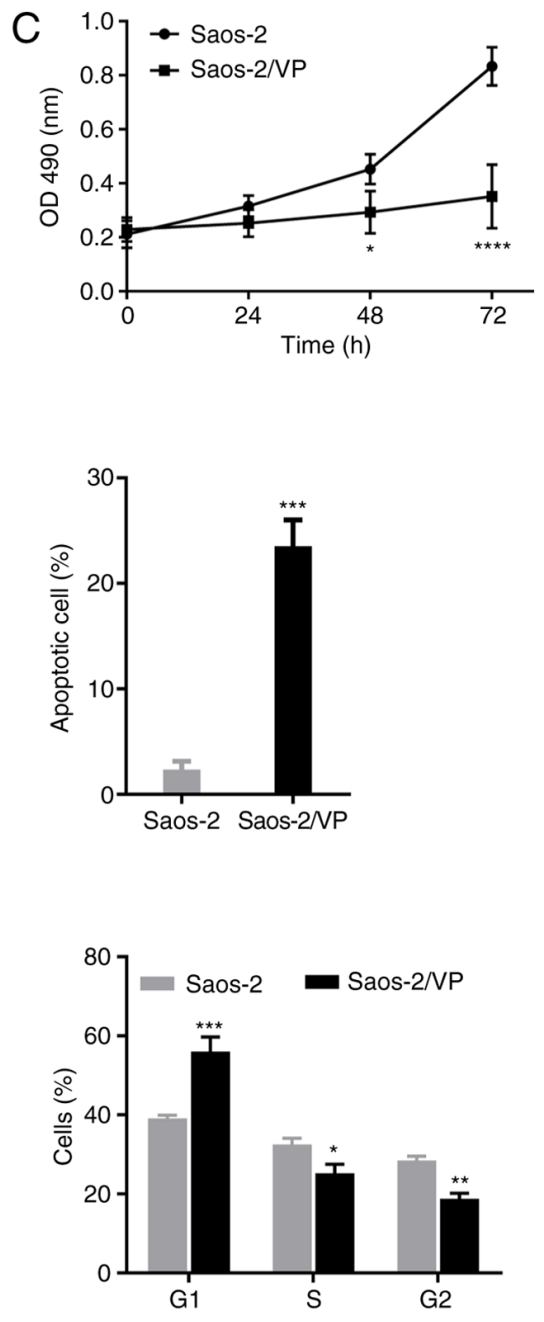

Figure 2. VPinhibits the expression of YAP1 and TEAD1 and regulates the viability, apoptosis and cell cycle progression of Saos-2 cells. (A) Western blot analysis was conducted to evaluate the protein expression of YAP1 and TEAD1 in Saos-2 cells treated with various concentrations of VP. (B) Co-immunoprecipitation assay was performed to detect the interaction between YAP1 and TEAD1 in Saos-2 cells with or without VP treatment. (C) MTT assay was performed to analyze the proliferation of Saos-2 cells with or without VP treatment. (D) VP promoted apoptosis of Saos-2 cells. (E) Flow cytometry was performed to analyze the cell cycle distribution. All experiments were performed in triplicate. ${ }^{*} \mathrm{P}<0.05,{ }^{* * *} \mathrm{P}<0.01,{ }^{* * *} \mathrm{P}<0.001$ and ${ }^{* * * *} \mathrm{P}<0.0001$ vs. Saos-2. YAP1, yes-associated protein 1; TEAD1, TEA domain transcription factor 1; VP, verteporfin; OD, optical density; IB, immunoblot; IP, immunoprecipitation.

Fig. 3C and D, on day 28 post-inoculation, significantly lower tumor volumes and weights were observed in the VP-treated mice compared with those in the saline-treated mice (volume, $449.521 \pm 151.391$ vs. $1,299.008 \pm 292.319 \mathrm{~mm}^{3}$; weight, $0.785 \pm 0.073$ vs. $1.718 \pm 0.244 \mathrm{~g})$.

CYR61 overexpression antagonizes the inhibitory effects of VP. To determine how VP acts on the Hippo signaling pathway in osteosarcoma, the present study examined the expression of the downstream target genes of TEAD1, including DYN3, PTGS2, LOX, BDNF, CTGF and CYR61, in Saos-2 cells treated with VP. As presented in Fig. 4A and B, VP administration led to a notable decrease in the mRNA and protein expression levels of PTGS2, LOX, CTGF and CYR61 compared with those in the control group, among which CYR61 displayed the most significant changes.

The association between the inhibitory effects of VP on osteosarcoma and CYR61 was further analyzed. For this purpose, the present study established an oeCYR61 cell line by infecting Saos-2 cells with CYR61 overexpressing lentivirus. The results of the RT-qPCR and western blot assays demonstrated that CYR61 expression levels were significantly increased in the oeCYR61 cells compared with those in the control group (Fig. 4C). The present study next investigated whether CYR61 overexpression affected the inhibitory effects of VP on Saos-2 cells. As demonstrated in Fig. 4D, compared with that in the control group, significantly reduced cell viability was observed in the oeCYR61 $+\mathrm{VP}$ and VP alone group, although the oeCYR61 + VP group exhibited a lower reduction than the VP alone group. Similarly, CYR61 overexpression attenuated the apoptosis in Saos-2 cells treated with VP, as indicated by the decreased apoptotic rate from $27.5 \%$ in the VP alone group to $16.4 \%$ in the oeCYR61 + VP group (Fig. 4E). In addition, CYR61 overexpression led to an increase in the number of cells in the $\mathrm{G} 2$ phase from $18.8 \%$ in the VP group to $28.8 \%$ in the oeCYR61 + VP group and decreases in the percentages of cells in the G1 and S phases from 60.0 and $25.2 \%$ in the VP group to 45.7 and $21.8 \%$ in the oeCYR61 + VP group, respectively (Fig. 4F). 

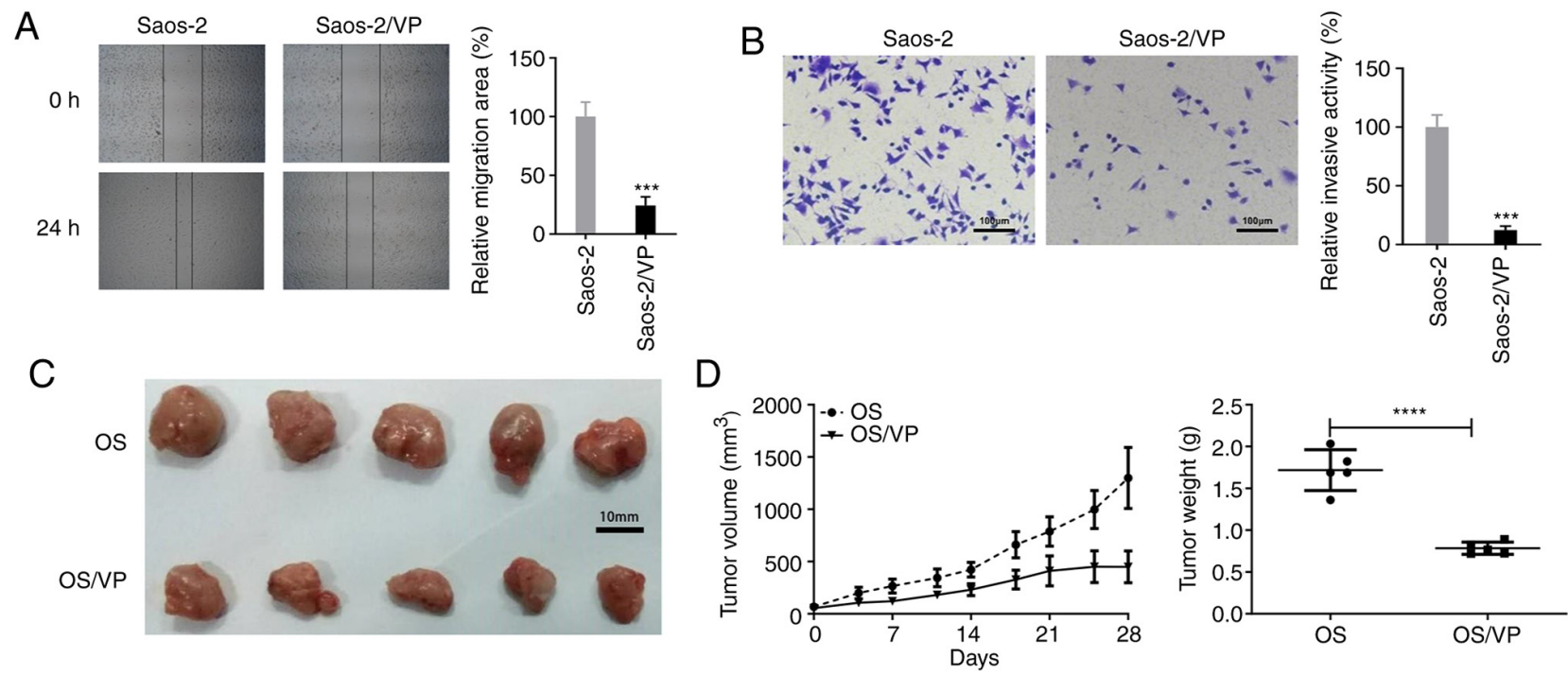

Figure 3. VP suppresses tumor cell migration and invasion as well as tumor growth. (A) Wound healing assay was conducted to detect the effects of VP on Saos-2 cell migration. Magnification, $\mathrm{x} 100 .{ }^{* * *} \mathrm{P}<0.001$ vs. Saos-2. (B) Transwell assays were performed to detect the effects of VP on the invasive ability of Saos-2 cells. Scale bar, $100 \mu \mathrm{m}$; magnification, $\mathrm{x} 100 .{ }^{* * * *} \mathrm{P}<0.001$ vs. Saos-2. (C) VP inhibited the growth of Saos-2 xenograft tumors in nude mice compared with that in the untreated group. (D) Changes in the volume and weight of OS xenograft tumors in mice following VP treatment. All experiments were performed in triplicate. ${ }^{* * * *} \mathrm{P}<0.0001$ vs. OS group. VP, verteporfin; OS, osteosarcoma.

\section{Discussion}

The current study presented evidence that VP reduced cell viability, migration and invasion, induced cell cycle arrest at the G1 phase and promoted apoptosis in osteosarcoma cells by downregulating the expression of the Hippo signaling pathway proteins YAP1 and TEAD1. To demonstrate the effects of VP on osteosarcoma, the present study comparatively analyzed the expression levels of YAP1 and TEAD1 in osteosarcoma tissues and paired tumor-adjacent tissues, as well as in a normal human osteoblast cell line (NHOst) and osteosarcoma cell lines (U2OS, HOS and Saos-2). The results demonstrated that YAP1 and TEAD1 were highly expressed in osteosarcoma cells and tissues, especially in Saos-2 cells. Consistently, previous studies have demonstrated that YAP1 is implicated in tumorigenesis, facilitating the progression of a number of types of cancer including gastric, liver and peritoneal cancer (24-26), whereas TEAD1 expression is upregulated in various types tumor such as medulloblastoma, renal and gastric cancer, suggesting a tumor-promoting role for TEADs $(27,28)$.

The Hippo signaling pathway was first identified in the Drosophila and was subsequently determined to be conserved in humans (29). Dysfunction of the Hippo signaling pathway has been reported to be involved in the occurrence of various types of cancer, such as cervical squamous cell carcinoma and mesothelioma $(30,31)$. YAP1 and TEAD1 are the major components of the Hippo signaling pathway in osteosarcoma (32). Upon activation, YAP1 and TAZ form a heterodimer that enters the nucleus to activate TEAD1, initiating gene transcription (33). Chai et al (12) have reported that the representative dysregulated profile of Hippo signaling in osteosarcoma is the formation of YAP1/TEAD1 complex.

VP is a drug predominantly used for macular degeneration and choroidal neovascularization that potently restrains the transcriptional activity of YAP1 (34). A study by Zhang et al (16) has demonstrated that VP inhibits tumor growth in a colorectal cancer model. To determine whether VP had an effect on and osteosarcoma, the present study conducted a series of experiments and revealed that VP exerted tumor-suppressive effects by reducing the viability, migration and invasion of Saos-2 cells. Subsequently, the inhibitory function of VP on YAP1 was assessed by detecting the expression levels of YAP1 and the interaction between YAP1 and its downstream target TEAD1. Notably, VP downregulated YAP1 expression levels and inhibited the interaction between YAP1 and TEAD1 in Saos-2 cells. Taken together, these data suggested that VP may potentially constrain the development of osteosarcoma by targeting the Hippo signaling pathway.

The results of the present study also demonstrated that CYR61 overexpression mitigated the inhibitory effects of VP on osteosarcoma progression. CYR61, which is a downstream target of the Hippo pathway, acts as one of the oncoproteins involved in tumor migration, adhesion and angiogenesis (35). Zhou et al (36) have identified an inhibitory function of downregulated CYR61 in melanoma, whereas Xie et al (37) have reported an association between high CYR61 expression levels and a poor prognosis in colorectal cancer. Notably, statins can target CYR61 and downregulate its expression in osteosarcoma cells (38). In the current study, VP treatment led to a marked decrease in CYR61 expression levels in osteosarcoma cells. It has been demonstrated that suppression of the formation of the YAP1/TEAD1 complex leads to the abnormal termination of signal transduction, reducing the expression levels of YAP1 downstream targets (12). The results of the present study revealed that VP decreased the expression levels of CYR61, while inhibiting cell proliferation in osteosarcoma, which mainly manifested as the cell cycle arrest, reduced cell migration and an increased apoptotic rate compared with those observed in the control group. Overexpression of CYR61 mitigated the cell cycle arrest and apoptosis, thus relieving the inhibitory effects of VP on osteosarcoma cells. Based on these observations, we hypothesized that CYR61 may act as 
A

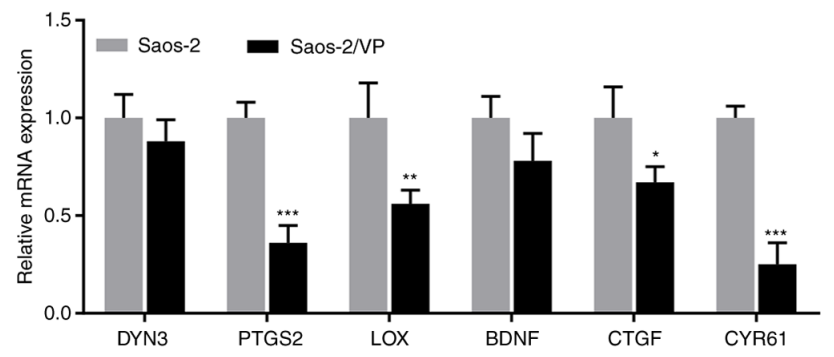

B
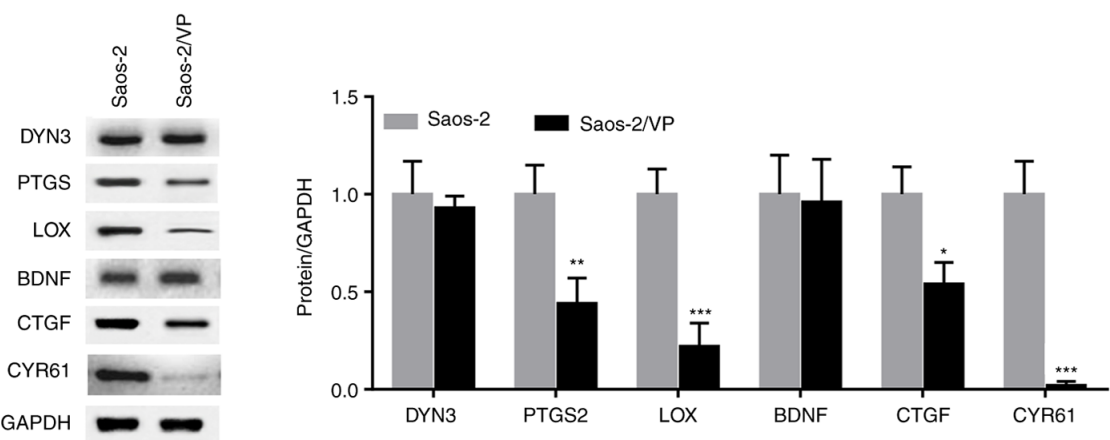

C
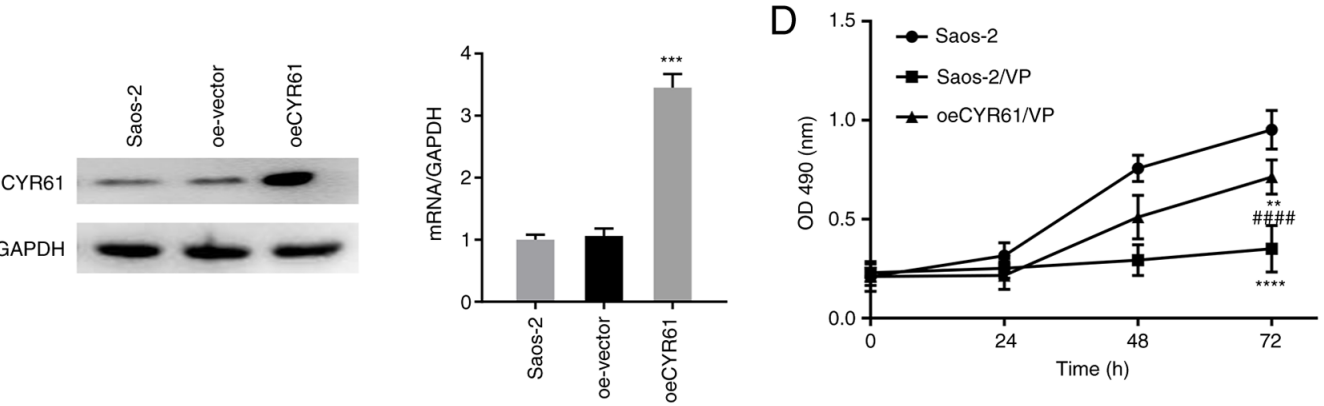

E
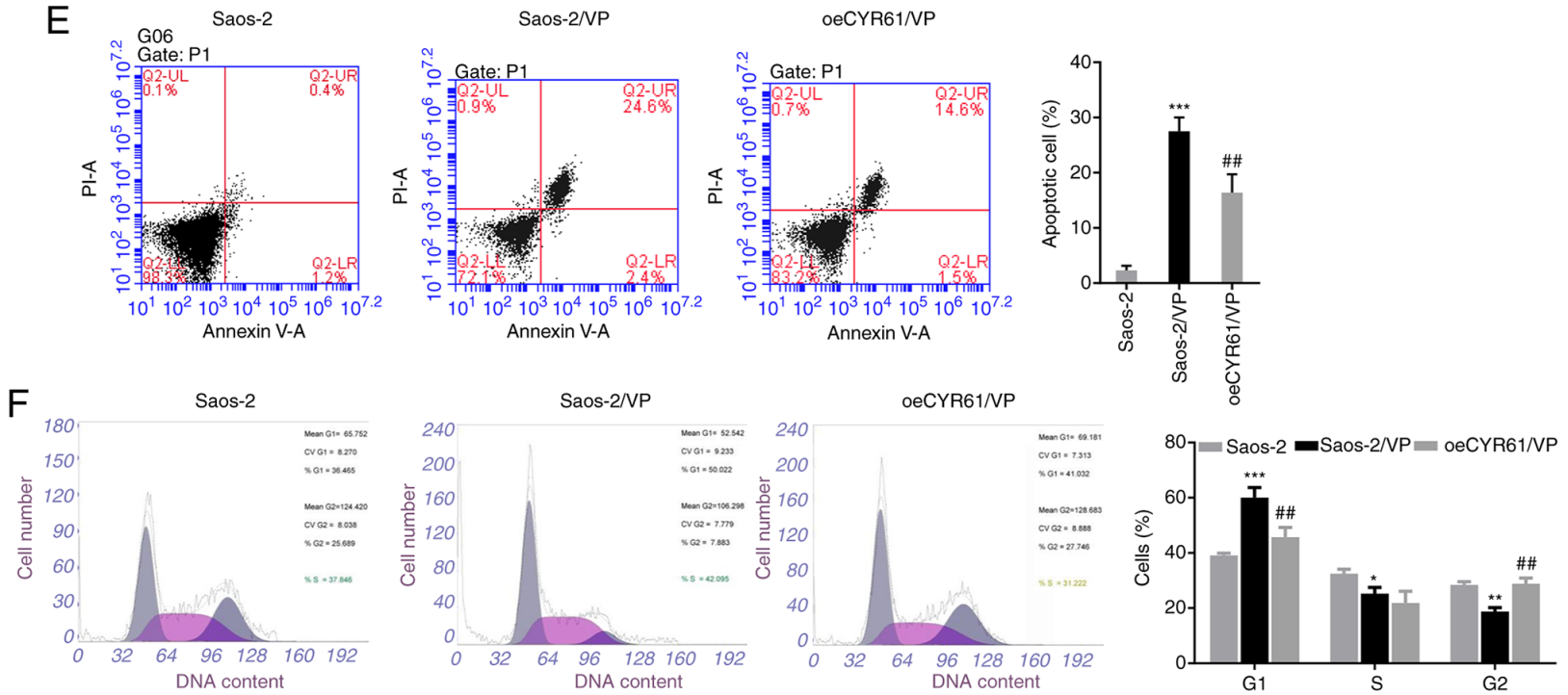

Figure 4. CYR61 overexpression partially reverses the inhibitory effects of VP on osteosarcoma cells. (A) RT-qPCR and (B) western blot analyses were performed to detect the mRNA and protein expression levels of the downstream targets of the Hippo signaling pathway, respectively. (C) Saos-2 cells were infected with the oeCYR61 lentivirus, and RT-qPCR and western blot analyses were conducted to determine the expression levels of CYR61 in the infected cells. (D) The effects of CYR61 overexpression on the proliferation of Saos-2 cells treated with VP were evaluated using the MTT assay. (E) Apoptosis and (F) cell cycle distribution in the oeCYR61 cells were analyzed by flow cytometry. All experiments were performed in triplicates. ${ }^{*} \mathrm{P}<0.05,{ }^{* *} \mathrm{P}<0.01,{ }^{* * * *} \mathrm{P}<0.001$ and ${ }^{* * * * *} \mathrm{P}<0.0001$ vs. Saos-2; ${ }^{\# \#} \mathrm{P}<0.01,{ }^{\# \# \# \#} \mathrm{P}<0.0001$ vs. Saos-2/VP. CYR61, cysteine-rich protein 61; VP, verteporfin; RT-qPCR, reverse transcription-quantitative PCR; oe, overexpression; PI, propidium iodide; PTGS2, prostaglandin-endoperoxide synthase 2; LOX, lysyl oxidase; BDNF, brain-derived neurotrophic factor; CTGF, connective tissue growth factor; DYN3, dynamin 3.

a key molecule in the formation and development of osteosarcoma. In addition, VP caused significant downregulation in the mRNA and protein expression levels of the oncogenic targets of YAP (PTGS2, LOX and CTGF) in Saos-2 cells in 
the present study. Consistently, Chai et al (12) have demonstrated that knockdown of TEAD1 led to a reduction in the expression of PTGS2, LOX and CTGF at mRNA levels, suggesting that PTGS2, LOX and CTGF may contribute to the oncogenic effects of TEAD1. The present study had several limitations, such as the lack of luciferase activity assay to further confirm the enhancement of the transcriptional activity of YAP1/TEAD1 complex in osteosarcoma tissues, which requires further study in the future.

In conclusion, VP may inhibit the development of osteosarcoma by downregulating the expression of the Hippo signaling pathway downstream target genes YAP1, TEAD1 and CYR61. In addition, the results of the present study demonstrated that CYR61 overexpression relieved the inhibitory effects of VP on osteosarcoma, suggesting that CYR61 may potentially serve as a therapeutic target for osteosarcoma.

\section{Acknowledgements}

Not applicable.

\section{Funding}

No funding was received.

\section{Availability of data and materials}

The datasets used and/or analyzed during the current study are available from the corresponding author on reasonable request.

\section{Authors' contributions}

XY designed the study and revised the manuscript. YX and CJ conducted the experiments. ZM and LJ collected and analyzed the data. XY and LJ confirmed the authenticity of all the raw data. All authors contributed to writing the manuscript. All authors read and approved the final manuscript.

\section{Ethics approval and consent to participate}

All patients and their guardians signed written informed consent and the procedure was approved by the Hospital Ethics Committee of the First People's Hospital of Wenling (approval no. KY-2019-2001-01). The protocols of animal experiments were approved by the Wenzhou Medical University Animal Policy and Welfare Committee (approval no. wydw2019-0616).

\section{Patient consent for publication}

Not applicable.

\section{Competing interests}

The authors declare that they have no competing interests.

\section{References}

1. Shi K, Wang SL, Shen B, Yu FQ, Weng DF and Lin JH: Clinicopathological and prognostic values of fibronectin and integrin $\alpha v \beta 3$ expression in primary osteosarcoma. World J Surg Oncol 17: 23, 2019.
2. Lokau J, Schoeder V and Garbers C: The role of interleukin-11 in osteosarcoma. Pathologe 41: 163-167, 2020 (In German).

3. Mason NJ: Comparative immunology and immunotherapy of canine osteosarcoma. Adv Exp Med Biol 1258: 199-221, 2020.

4. Lin CH, Guo Y, Ghaffar S, McQueen P, Pourmorady J, Christ A, Rooney K, Ji T, Eskander R, Zi X and Hoang BH: Dkk-3, a secreted wnt antagonist, suppresses tumorigenic potential and pulmonary metastasis in osteosarcoma. Sarcoma 2013: 147541, 2013.

5. Czarnecka AM, Synoradzki K, Firlej W, Bartnik E, Sobczuk P, Fiedorowicz M, Grieb P and Rutkowski P: Molecular biology of osteosarcoma. Cancers (Basel) 12: 2130, 2020.

6. Kansara M, Teng MW, Smyth MJ and Thomas DM: Translational biology of osteosarcoma. Nat Rev Cancer 14: 722-735, 2014.

7. Han G, Wang Y and Bi W: C-Myc overexpression promotes osteosarcoma cell invasion via activation of MEK-ERK pathway. Oncol Res 20: 149-156, 2012.

8. Maire G, Martin JW, Yoshimoto M, Chilton-MacNeill S, Zielenska $M$ and Squire JA: Analysis of miRNA-gene expression-genomic profiles reveals complex mechanisms of microRNA deregulation in osteosarcoma. Cancer Genet 204: 138-146, 2011.

9. Sun ZY, Jian YK, Zhu HY and Li B: lncRNAPVT1 targets miR-152 to enhance chemoresistance of osteosarcoma to gemcitabine through activating c-MET/PI3K/AKT pathway. Pathol Res Pract 215: 555-563, 2019.

10. Sun Y, Jiang T, Jia Y, Zou J, Wang X and Gu W: LncRNA MALAT1/miR-181a-5p affects the proliferation and adhesion of myeloma cells via regulation of Hippo-YAP signaling pathway. Cell Cycle 18: 2509-2523, 2019.

11. Johnson R and Halder G: The two faces of Hippo: Targeting the Hippo pathway for regenerative medicine and cancer treatment. Nat Rev Drug Discov 13: 63-79, 2014.

12. Chai J, Xu S and Guo F: TEAD1 mediates the oncogenic activities of Hippo-YAP1 signaling in osteosarcoma. Biochem Biophys Res Commun 488: 297-302, 2017.

13. Chan LH, Wang W, Yeung W, Deng Y, Yuan P and Mak KK: Hedgehog signaling induces osteosarcoma development through Yap1 and H19 overexpression. Oncogene 33: 4857-4866, 2014.

14. Kyriazoglou A, Liontos M, Zakopoulou R, Kaparelou M, Tsiara A, Papatheodoridi AM, Georgakopoulou R and Zagouri F: The role of the hippo pathway in breast cancer carcinogenesis, prognosis, and treatment: A systematic review. Breast Care (Basel) 16: 6-15, 2021.

15. Zanconato F, Forcato M, Battilana G, Azzolin L, Quaranta E, Bodega B, Rosato A, Bicciato S, Cordenonsi M and Piccolo S: Genome-wide association between YAP/TAZ/TEAD and AP-1 at enhancers drives oncogenic growth. Nat Cell Biol 17: 1218-1227, 2015.

16. Zhang H, Ramakrishnan SK, Triner D, Centofanti B, Maitra D, Győrffy B, Sebolt-Leopold JS, Dame MK, Varani J, Brenner DE, et al: Tumor-selective proteotoxicity of verteporfin inhibits colon cancer progression independently of YAP1. Sci Signal 8: ra98, 2015

17. Chen WS, Cao Z, Krishnan C and Panjwani N: Verteporfin without light stimulation inhibits YAP activation in trabecular meshwork cells: Implications for glaucoma treatment. Biochem Biophys Res Commun 466: 221-225, 2015.

18. Andrade D, Mehta M, Griffith J, Panneerselvam J, Srivastava A, Kim TD, Janknecht R, Herman T, Ramesh R and Munshi A: YAP1 inhibition radiosensitizes triple negative breast cancer cells by targeting the DNA damage response and cell survival pathways. Oncotarget 8: 98495-98508, 2017.

19. Pan W, Wang Q, Zhang Y, Zhang N, Qin J, Li W, Wang J, Wu F, Cao L and Xu G: Verteporfin can reverse the paclitaxel resistance induced by YAP over-expression in HCT-8/T cells without photoactivation through inhibiting YAP expression. Cell Physiol Biochem 39: 481-490, 2016.

20. Lu Q, Lv G, Kim A, Ha JM and Kim S: Expression and clinical significance of extracellular matrix metalloproteinase inducer, EMMPRIN/CD147, in human osteosarcoma. Oncol Lett 5: 201-207, 2013.

21. Livak KJ and Schmittgen TD: Analysis of relative gene expression data using real-time quantitative PCR and the 2(-Delta Delta C(T)) method. Methods 25: 402-408, 2001.

22. Wang DY, Wu YN, Huang JQ, Wang W, Xu M, Jia JP, Han G, Mao BB and Bi WZ: Hippo/YAP signaling pathway is involved in osteosarcoma chemoresistance. Chin J Cancer 35: 47, 2016.

23. Zanconato F, Cordenonsi M and Piccolo S: YAP/TAZ at the roots of cancer. Cancer Cell 29: 783-803, 2016. 
24. Sun D, Wang Y, Wang $H$ and Xin Y: The novel long non-coding RNA LATS2-AS1-001 inhibits gastric cancer progression by regulating the LATS2/YAP1 signaling pathway via binding to EZH2. Cancer Cell Int 20: 204, 2020.

25. Liu YP, Pan LL and Kong CC: Stathmin 1 promotes the progression of liver cancer through interacting with YAP1. Eur Rev Med Pharmacol Sci 24: 7335-7344, 2020.

26. Ajani JA, Xu Y, Huo L, Wang R, Li Y, Wang Y, Pizzi MP, Scott A, Harada K, Ma L, et al: YAP1 mediates gastric adenocarcinoma peritoneal metastases that are attenuated by YAP1 inhibition. Gut 70: 55-66, 2021.

27. Zhou Y, Huang T, Cheng AS, Yu J, Kang W and To KF: The TEAD family and its oncogenic role in promoting tumorigenesis. Int J Mol Sci 17: 138, 2016

28. Zhou Y, Huang T, Zhang J, Wong CC, Zhang B, Dong Y, Wu F, Tong JHM, Wu WKK, Cheng ASL, et al: TEAD1/4 exerts oncogenic role and is negatively regulated by miR-4269 in gastric tumorigenesis. Oncogene 36: 6518-6530, 2017.

29. Zheng Y and Pan D: The hippo signaling pathway in development and disease. Dev Cell 50: 264-282, 2019.

30. Wang Y, Xu X, Maglic D, Dill MT, Mojumdar K, Ng PK, Jeong KJ, Tsang YH, Moreno D, Bhavana VH, et al: Comprehensive molecular characterization of the hippo signaling pathway in cancer. Cell Rep 25: 1304-1317.e5, 2018.

31. Yimlamai D, Fowl BH and Camargo FD: Emerging evidence on the role of the Hippo/YAP pathway in liver physiology and cancer. J Hepatol 63: 1491-1501, 2015

32. Plouffe SW, Hong AW and Guan KL: Disease implications of the Hippo/YAP pathway. Trends Mol Med 21: 212-222, 2015.

33. Hong L, Cai Y, Jiang M, Zhou D and Chen L: The Hippo signaling pathway in liver regeneration and tumorigenesis. Acta Biochim Biophys Sin (Shanghai) 47: 46-52, 2015.
34. Liu K, Du S, Gao P and Zheng J: Verteporfin suppresses the proliferation, epithelial-mesenchymal transition and stemness of head and neck squamous carcinoma cells via inhibiting YAP1. J Cancer 10: 4196-4207, 2019.

35. Park MH, Kim AK, Manandhar S, Oh SY, Jang GH, Kang L, Lee DW, Hyeon DY, Lee SH, Lee HE, et al: CCN1 interlinks integrin and hippo pathway to autoregulate tip cell activity. Elife 8: e46012, 2019.

36. Zhou X, Rao Y, Sun Q, Liu Y, Chen J and Bu W: Long noncoding RNA CPS1-IT1 suppresses melanoma cell metastasis through inhibiting Cyr61 via competitively binding to BRG1. J Cell Physiol 234: 22017-22027, 2019.

37. Xie L, Song X, Lin H, Chen Z, Li Q, Guo T, Xu T, Su T, Xu M, Chang X, et al: Aberrant activation of CYR61 enhancers in colorectal cancer development. J Exp Clin Cancer Res 38: 213, 2019.

38. Fromigue O, Hamidouche Z, Vaudin P, Lecanda F, Patino A, Barbry P, Mari B and Marie PJ: CYR61 downregulation reduces osteosarcoma cell invasion, migration, and metastasis. J Bone Miner Res 26: 1533-1542, 2011.

This work is licensed under a Creative Commons Attribution-NonCommercial-NoDerivatives 4.0 International (CC BY-NC-ND 4.0) License. 